\title{
A Case of Mucocutaneous Colloid Milium in an Adult Filipino Female with Chronic Kidney Disease
}

\author{
Shahara Abalos-Babaran ${ }^{1}$ and Eileen Liesl A. Cubillan ${ }^{2}$ \\ ${ }^{1}$ Section of Dermatology, Department of Medicine, Philippine General Hospital, University of the Philippines Manila \\ ${ }^{2}$ Section of Dermatology, Department of Medicine, College of Medicine and Philippine General Hospital, University of the Philippines Manila
}

\begin{abstract}
A 65-year-old female with chronic kidney disease (CKD) presented with a six-month history of asymptomatic translucent skin-colored and yellow plaques on the face. Similar yellow papules and nodules were noted in the oral cavity and eyelid margins. Biopsies were taken from the face and oral cavity showed homogeneous eosinophilic deposits with clefts in the dermis consistent with colloid milium. The patient was treated with fractional and surgical carbon dioxide $\left(\mathrm{CO}_{2}\right)$ laser with improvement after one session.
\end{abstract}

Key Words: colloid milium, $\mathrm{CO}_{2}$ laser, chronic kidney disease

\section{INTRODUCTION}

Colloid milium is a rare disorder clinically characterized by firm, round, translucent, skin-colored to yellow papules in sun-exposed areas such as face and neck. But there are few case reports in non-sun-exposed areas namely the inner surface of the ears ${ }^{1}$, penile skin, ${ }^{2}$ eyelids, ${ }^{3,4}$ and oral cavity. ${ }^{5}$ There are also reports of coexistence with vitamin C deficiency, ${ }^{6}$ trichinosis ${ }^{7}$, and beta-thalassemia. ${ }^{8}$ But, no disease association has been definitely established. This is the first case of adult colloid milium with cutaneous, oral, and eyelid margins lesions associated with chronic kidney disease.

\section{CLINICAL CASE}

A 65-year-old female presented with a six-month history of multiple asymptomatic skin-colored to yellow translucent papules and plaques on the face. Some lesions expressed gelatinous material with hemorrhage upon gentle pressure. No medications were applied. Numerous nonpainful yellowish to white papules and plaques were also noted on the tongue spreading to the buccal mucosa.

The patient is a diagnosed case of chronic kidney disease secondary to chronic glomerulonephritis. She was maintained on oral febuxostat, ferrous sulfate, folic acid, sodium bicarbonate and keto-analogue with good compliance and on regular follow up at our institution's nephrology section. She previously worked as a shrimp paste vendor for 40 years at an open public market and was greatly exposed to sunlight.

On physical examination, there were multiple, discreet, firm skin-colored to yellow, round shiny papules and plaques with cobblestone appearance mostly on the periorificial areas (Figures $1 \mathrm{~A}, \mathrm{C}, \mathrm{E}$ ). Hemorrhage was noted over some 

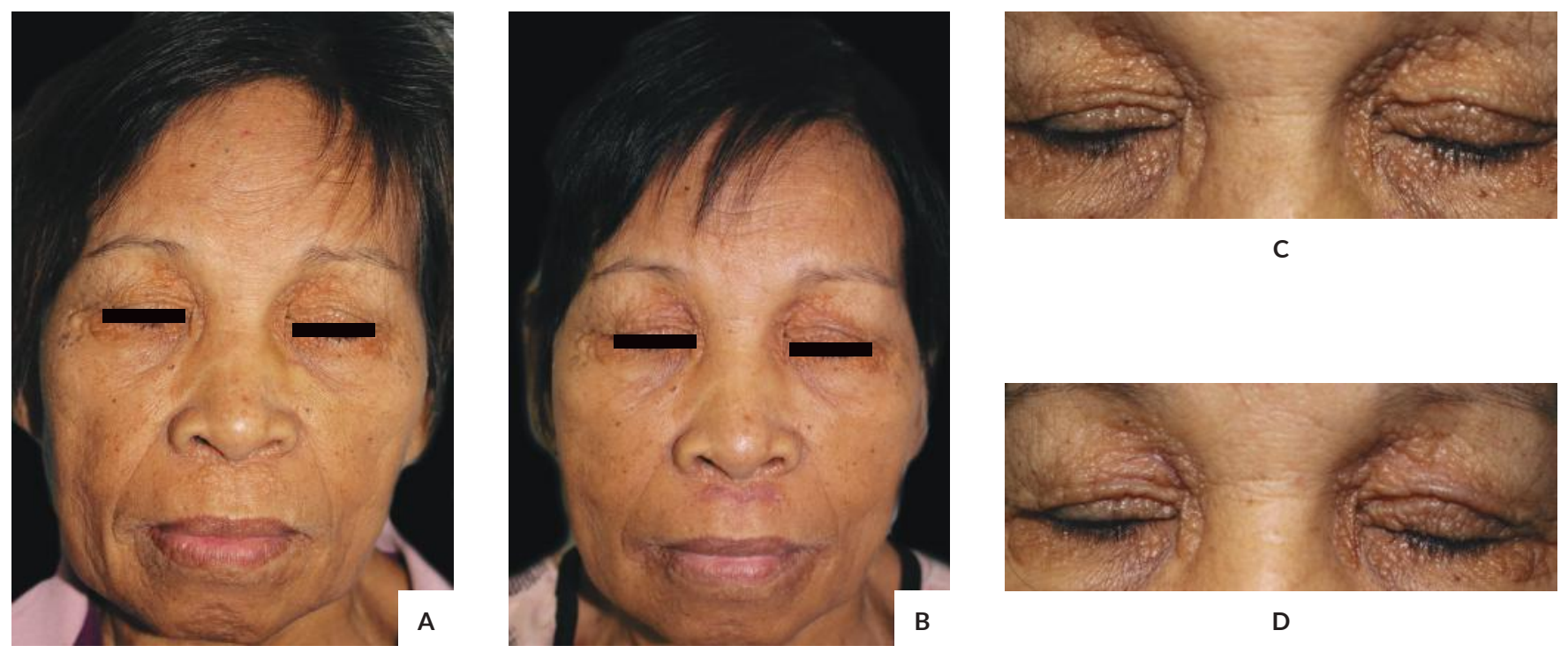

C

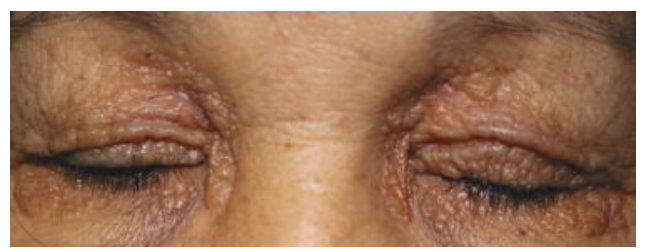

D
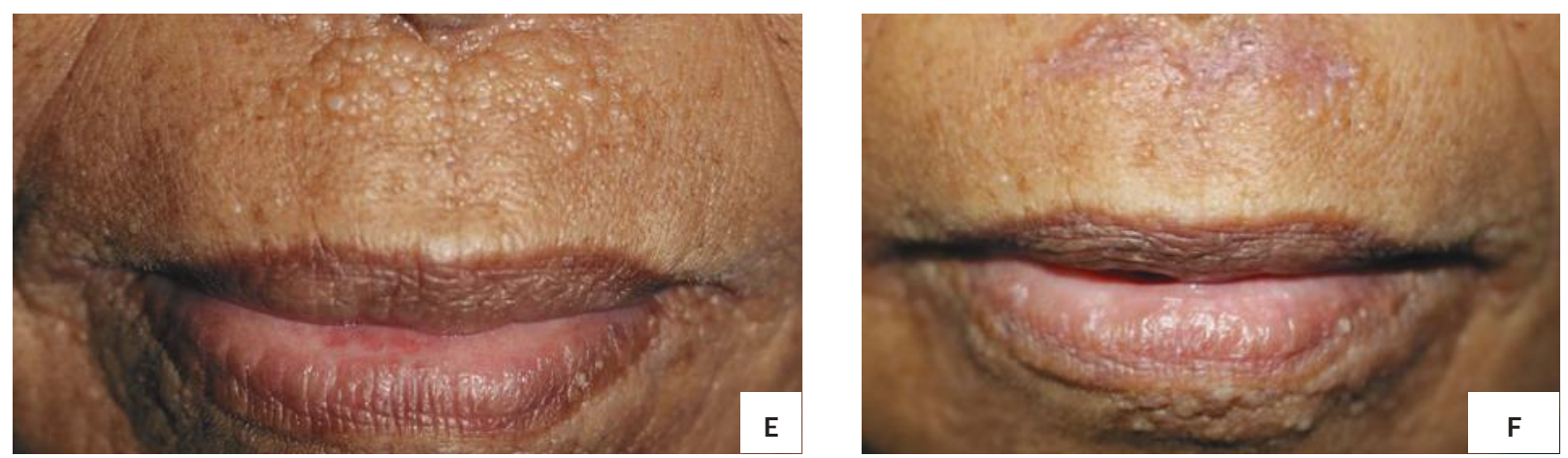

Figure 1. Multiple skin-colored and yellow papules on the face before (A) and after (B) treatment with $\mathrm{CO}_{2}$ laser. Closer view of the periorbital area before (C) and after (D) treatment with fractionated $\mathrm{CO}_{2}$ laser showing decreased thickness of plaque. Closer view of the perioral area before (E) and after (F) treatment with surgical $\mathrm{CO}_{2}$ laser showing fully ablated lesions.

areas on the left periorbital plaque. Similar translucent skin colored papules were also observed on the bilateral upper and lower eyelid (Figures $2 \mathrm{~A}$ and $\mathrm{B}$ ) growing on the base of the eyelashes and concha of the bilateral ears. Examination of the oral cavity showed similar discreet yellow soft grouped papules, plaques and nodules on the angle and vermillion border of the lip, labial mucosa, buccal mucosa and tongue as shown in Figures $2 \mathrm{C}$.

Three millimeter punch biopsies of skin lesion and several oral lesions were done. On histopathologic examination, the skin-colored plaque over the left upper periorbital area showed dermal deposits of homogenous, amorphous, pale eosinophilic material with clefts along which fibroblasts are aligned. The overlying epidermis is atrophic with a thin grenz zone separating this from the dermal deposits. There is marked solar elastosis in the underlying dermis with very sparse inflammatory infiltrate surrounding the eosinophilic deposits. Similarly, the histologic sections of the specimens taken from the yellow plaque on the lower labial mucosa and a yellow nodule on the dorsum of the tongue revealed islands of homogeneous pink deposits with multiple fissures spanning the whole dermis. These histopathologic findings confirm the clinical diagnosis of colloid milium.

There was slight hypertriglyceridemia $(225.45 \mathrm{mg} /$ dl), elevated creatinine (202. 2 umol/L), high blood urea nitrogen (BUN) $(8.6 \mathrm{mmol} / \mathrm{L})$, low hemoglobin $(88 \mathrm{mg} /$ $\mathrm{dL}$ ) and slight albuminuria $(+1)$ which can be all attributed to the concomitant CKD.

The patient was advised to apply tretinoin $0.025 \%$ cream at night to whole face and sunscreen SPF 30, broad spectrum cream. Ablation of the facial lesions using $\mathrm{CO}_{2}$ laser was done. Fractional mode [active mode, size (square), energy $300 \mathrm{~mJ}$, depth level $5 \mathrm{um}$ ) was used on the periorbital plaques since this area has thinner skin and is prone to development of purpura while the surgical mode was chosen for the perinasal and perioral lesions. After two weeks, there was minimal thinning of the plaques over the upper periorbital area with complete reepithelization as shown in Figure 1 (D). In contrast, a better outcome was achieved with a surgical laser on the perinasal and 

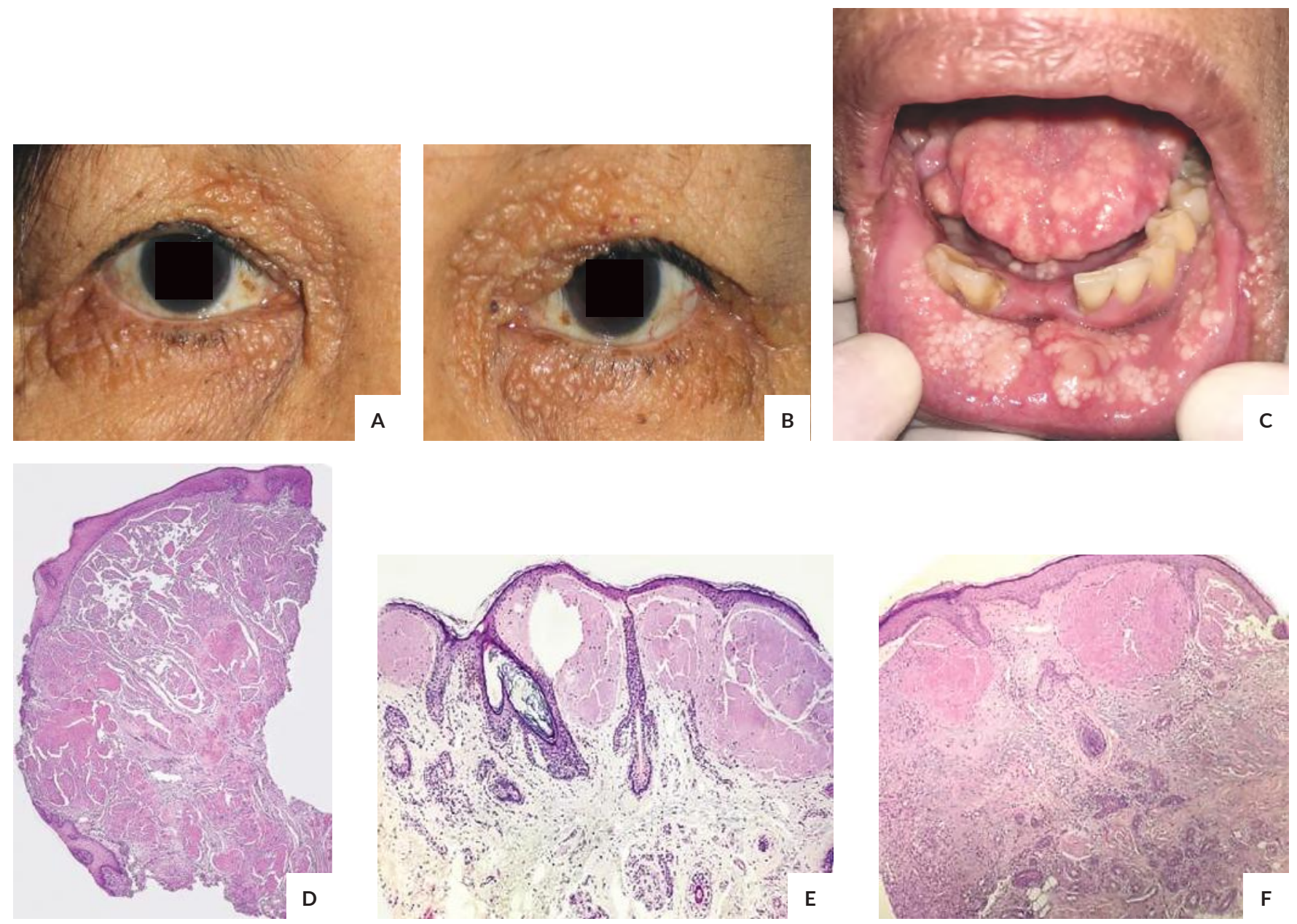

Figure 2. Multiple translucent papules on the base of the right (A) and left (B) upper and lower eyelid margins. Hemorrhagic papules seen on the periorbital area (B). Similar multiple white-yellow papules, plaques and nodules noted on the oral cavity. Biopsy $(H \& E, x 10)$ from the tongue nodule showing islands of amorphous eosinophilic material with fissures spanning the whole dermis consistent with colloid milium (D). Biopsy (H\&E, $x 40)$ of the left periorbital plaque pretreatment (E) showing atrophic epidermis with homogeneous eosinophilic deposits with clefts in the upper dermis. (F) - Posttreatment biopsy (H\&E, x40) showed fewer, smaller and less circumscribed dermal deposits.

perioral areas as most lesions were fully ablated as seen in Figure 1 (F). A post-treatment biopsy was taken from the left upper periorbital lesion showing fewer, smaller and less circumscribed dermal deposits compared to before shown in Figure $2(\mathrm{~F})$.

\section{DISCUSSION}

\section{Pathophysiology}

The exact pathophysiology of this disease is not yet fully elucidated, but there is a consensus among authors that sunlight exposure plays a significant role. There are several speculations about the origin of the colloid material.

The widely accepted theory implicates the actinic degeneration of elastic fibers as the origin of colloid. Chronic UV exposure may destroy the structure of elastin directly, disturb the synthetic process of elastin in fibroblasts hence abnormal elastic fibers may be produced or incite the release of proteolytic enzymes. ${ }^{9}$ This is supported by an immunohistochemistry study which showed that colloid and elastic fibers exhibit similar microfibrillar proteins with no hydroxyproline. ${ }^{10}$ To add, electron microscopy revealed the presence of normal and degenerating elastic fibers within and around the colloid ${ }^{8}$ which are degraded into a granulo-fibrillar substance identical to the colloid material. ${ }^{9}$

Meanwhile, it was suggested that colloid originates from the actinic damage of preexisting collagen, or by de novo production of abnormal dermal fibroblasts. This is supported by the presence of degenerated collagen fibrils within the deposits and fibroblasts containing an amorphous substance indistinguishable from colloid. ${ }^{10}$ But the colloid material does not contain hydroxyproline or hydroxylysine, the characteristic amino acids comprising collagen rendering this as the less likely origin of colloid. 
Lastly, it was suggested that chronic sunlight exposure might induce changes in the walls of the dermal blood vessels, allowing the diffusion of the blood proteins into the connective tissue until they reached their deposition place similar to amyloid. ${ }^{11}$ This was supported by the presence of capillaries with swollen walls within and around the colloid masses. But this blood borne origin of colloid was disputed as there was sparing of perivascular spaces, an absent phagocytic colloid in perivascular phagocytes and unremarkable immunoglobulin level of patients. ${ }^{10}$

\section{Histopathologic and ultramicroscopic findings}

The closest histologic differential to these dermal deposits is cutaneous amyloidosis as both have eosinophilic amorphous material surrounded by absent to limited inflammatory cellular infiltration. However, amyloidosis has smaller deposits within the widened dermal papillae in contrast to colloid milium which has larger deposits located on almost the upper half of the dermis. ${ }^{12}$ Moreover, stellate fibroblasts and melanophages are closely admixed with amyloid while few fibroblasts and notable lack of macrophages are seen within the colloid. Lastly, an atrophic epidermis usually lies above the colloid material in contrast to the normal or hyperplastic epidermis seen over the amyloid deposits. Colloid milium, unlike amyloidosis, do not stain for immunoglobulins, complement, or antikeratin antibodies. A definite diagnosis can be made with electron microscopy considered as the gold standard because only amyloid shows long, straight, non-branching, randomly oriented filaments ${ }^{3}$ while colloid has short, branching, wavy granulo-fibrillar appearance ${ }^{2}$ in a criss-cross pattern with a smaller diameter.

\section{Association with Other Diseases}

It was proposed that lesion could be related to excess iron observed in beta- thalassemia. Free iron may induce the formation of hydroxyl radical that causes peroxidation of membrane lipids and proteins and may result in a disturbance of the elastin metabolism and structural deterioration of elastic fibers. ${ }^{8}$

There are no previous case reports of colloid milium associated with CKD. Iron content may increase in patients with CKD especially in cases of supplementation to address anemia from hypoproliferative erythroid marrow dysfunction as in this case. ${ }^{13}$ Similarly, the increased iron might have incited the formation of free radical causing oxidant tissue injury (degeneration of elastic fibers). Another mechanism is the inherent high oxidative stress in CKD. ${ }^{14}$ Evidence suggest that there is failure of reactive oxygen species (ROS) clearance with declining renal function due to decreased serum glutathione peroxidase activity. To add, CKD is an inflammatory process in itself leading to widespread ROS activation. ${ }^{14}$ These ROS also cause protein peroxidation as mentioned earlier resulting to alteration of the elastic fiber integrity. This may explain the occurrence of lesions even in non-sun-exposed areas. Moreover, the improvement of colloid milium in a vitamin $\mathrm{C}$ deficient patient ${ }^{6}$ by oral supplementation may be explained by its antioxidant property as free radical scavenger together with glutathione. ${ }^{15}$

Meanwhile, a group of cutaneous disorders commonly associated with chronic renal disese is acquired perforating disorder. One of the postulated pathogenesis involves trauma to the skin caused by repetitive scratching from chronic pruritus resulting in transepidermal elimination of dermal material. ${ }^{16} \mathrm{~A}$ subtype is elastosis perforans serpiginosa (EPS) characterized by protrusions of altered elastic fibers through the dermis and epidermis. Similarly, colloid milium occurs as subepidermal deposits which may contain altered elastic fibers. Instead of being eliminated through the epithelium, the material accumulates with no drastic inflammatory reaction. This lack of foreign body reaction suggests that the material is inherently produced in the body thus, behaving as an inert substance. ${ }^{17}$

\section{Treatment}

Currently, there is still no standard therapy for colloid milium. Several procedures such as dermabrasion, curettage, cryotherapy, erbium laser resurfacing, photodynamic therapy and retinoic acid have been tried. However, no treatment has given reliably excellent results.

$\mathrm{Li}$ et al. first used of fractionated $\mathrm{CO}_{2}$ laser to treat these lesions, achieving clinical clearance and no recurrence at the three month follow up. ${ }^{18}$ In contrast, there was a minimal response to fractional $\mathrm{CO}_{2}$ laser in our case owing to the depth of lesions unreached by this mode. Meanwhile, a marked difference was observed using the surgical $\mathrm{CO}_{2}$ laser as most lesions were mostly ablated and achieved complete epithelization within two weeks.

\section{CONCLUSION AND RECOMMENDATION}

Colloid milium and amyloidosis can appear very similar clinically and histologically, except for a few distinguishing features. Electron microscopy, though not readily available, remains to be the gold standard of diagnosis in the background of a firm clinical picture.

Although cutaneous lesions of colloid milium are well documented, a reference to oral lesions and eyelid involvement are very few. To the best of our knowledge, this is the first documented case of adult colloid milium presenting with cutaneous, oral, and eyelid margins lesions in a patient with chronic kidney disease. Although no disease has been ascertained to have a definite association with this rare condition, iron overload and oxidative stress in CKD may incite tissue injury particularly elastic fiber alteration resulting to the onset of lesions especially in cases without a history of significant UV exposure.

This is the first documented use of surgical $\mathrm{CO}_{2}$ laser achieving excellent cosmetic result with minimal transient 
local side effects such as erythema, superficial erosion and burning sensation. Unfortunately, no follow up treatment sessions and monitoring were done due to the demise of the patient from complications of renal failure.

\section{Statement of authorship}

All authors approved the final version submitted.

\section{Authors disclosure}

All authors declared no conflicts of interest.

\section{Funding source}

This paper was funded by the authors. No external funding agency.

\section{REFERENCES}

1. Findlay GH, Morrison JGL, Simson IW. Exogenous ochronosis and pigmented colloid milium from hydroquinone bleaching creams. Br J Dermatol. 1975;93(6):613-22.

2. Preston PW, Orpin SD, Muc R, Zaki I. Penile colloid degeneration. Clin Exp Dermatol. 2006;31(5):674-6.

3. Safneck JR, Quinonez G, Wiens JJ. Adult colloid milium of the eyelid. Am J Ophthalmol. 1997;123(3):402-3.

4. Muzaffar W, Dar NR, Malik AM. Colloid milium of the upper eyelid margins. Ophthalmology. 2002;109(10):1944-6.

5. Ojha J, Bhattacharyya I, Islam NM, Wong F, Cohen DM. Colloid milium of the oral cavity: a rare presentation. Oral Surg Oral Med Oral Pathol Oral Radiol Endodontology. 2008;105(4):e34-8.
6. Way S. Colloid milium: a vitamin deficiency disease?. Arch Derm Syphilol. 1942;45(6):1148-55.

7. Okhremchuk I, Abed S, Nguyen AT, Brandone N, Morand J-J. Milium colloïde : une observation associée à une trichinose. Ann Pathol. 2016;36(2):130-3.

8. Giordano G, Ricci R, Froio E, Izzi G, Barone A, Boccaletti V. A case of colloid milium in patient with $\beta$ thalassaemia major. J Cutan Pathol. 2008;35(6):566-9.

9. Kobayashi H, Hashimoto K. Colloid and elastic fibre: ultrastructural study on the histogenesis of colloid milium. J Cutan Pathol. 1983;10(2):111-22.

10. Hashimoto K, Katzman RL, Kang AH, Kanzaki T. Electron microscopical and biochemical analysis of colloid milium. Arch Dermatol. 1975;111:49-59.

11. Zoon JJ, Jansen LH, Hovenkamp A. The nature of colloid milium. Br J Dermatol. 1955;67(6):212-7.

12. Rongioletti F, Barnhill R. Textbook of dermatopathology. In: 3rd ed. New York: McGraw-Hill; 2010. p. 378-9.

13. Nankivell BJ, Boadle RA, Harris DCH. Iron accumulation in human chronic renal disease. Am J Kidney Dis. 1992;20(6):580-4.

14. Meenakshi Sundaram SP, Nagarajan S, Manjula Devi AJ. Chronic kidney disease-effect of oxidative stress. Chin J Biol. 2014;2014:1-6.

15. Montecinos V, Guzmán P, Barra V, Villagrán M, Muñoz-Montesino C, Sotomayor K, et al. Vitamin C Function in Endothelial Cells. J Biol Chem. 2007;282(21):15506-15.

16. Rapini R. Perforating diseases. In: Bolognia JL, Jorizzo JL, \& Schaffer JV, eds Dermatology. 3rd ed. Philadelphia; 2012. p. 1600-4.

17. Hashimoto K, Miller F, Bereston ES. Colloid milium. Histochemical and electron microscopic studies. Arch Dermatol. 1972;105(5):684-94.

18. Li YL, Luo YJ, Wu Y, Li B, Gao XH, Chen HD, et al. Infraredinduced adult colloid milium treated with fractionated $\mathrm{CO}_{2}$ laser: Adult colloid milium treated with $\mathrm{CO}_{2}$ laser. Dermatol Ther 2014;27(2):68-70.

\section{The Acta Medica Philippina is now accepting} original scientific papers, review articles and case reports for its upcoming issues. Please follow the format for submission as indicated in the "Instructions to Authors" elsewhere in the journal. All papers received shall be properly acknowledged. For inquiries and submission of proposals, please e-mail us at actamedicaphilippina@yahoo.com 